\title{
Análise do funcionamento de dispositivos LoRa e LoraWAN na cidade de Caxias do Sul/RS
}

\author{
Acácio Chinato $^{1}$, Ricardo Garcia ${ }^{1}$, Joel da Silva ${ }^{1}$, Eduardo Carvalho ${ }^{1}$, \\ Marcelo de Lima ${ }^{1}$, Joao Vitor de Lima ${ }^{1}$, Samuel F. Ferrigo ${ }^{1}$ \\ ${ }^{1}$ Centro Universitário UNIFTEC \\ Caxias do Sul - RS - Brasil \\ \{acacio.oliveira,010207, joel_387, eduardo.dias, marcelo_de_lima, \\ joao.lima, samuelferrigo\}eacad.ftec.com.br
}

\begin{abstract}
Resumo. O LoRa é uma das tecnologias que oferece comunicação a longas distâncias com baixo consumo de energia para dispositivos IoT. Neste trabalho foram testados o alcance da transmissão e sua integração com a rede LoraWAN existente na cidade de Caxias do Sul/RS. Como resultados obtidos, chegou-se à distância máxima de 240 metros e foi possível conectar-se à rede LoraWAN somente através de um gateway alternativo.
\end{abstract}

\section{Introdução}

A Internet das Coisas (IoT) trouxe novas novas possibilidades de uso da computação. Algumas das possibilidades são o uso na medicina, nas aplicações militares, na automação residencial e nas cidades inteligentes [Avelar et al. 2010] [Ferrigo et al. 2017]. Neste último caso, diversos são os desafios: controle do transporte público, consumo de água e energia, segurança pública são alguns deles [Tomas 2014].

Com a proliferação dispositivos IoT em cidades, a necessidade de uma nova forma de comunicação de longa distância com baixo conusumo de energia [Augustin et al. 2016] é necessária. Uma das tecnologias que oferece isso é a LoRa. Comparado a outras tecnologias similares, como o SigFox, uma das vantagens que essa tecnolgia oferece é a possibilidade de comunicação dos dispositivos LoRa com aplicações conectadas à Internet, através do protocolo LoraWAN [Alliance 2015].

No presente trabalho foi buscado entender e testar os recursos desta nova tecnologia, como o alcance do sinal e o seu funcionamento na rede LoraWAN instalada na cidade de Caxias do Sul/RS. Para realização desses testes foram criados os ambientes descritos na Seção 3.

\section{As tecnologias LoRa e LoraWAN}

Acrônimo para "Long Range", o LoRa é um sistema de comunicação sem fio desenvolvido pela Lora Alliance. Utiliza diversas bandas de rádio que permitem a comunicação a longas distâncias com consumo mínimo de energia [Alliance 2015], promovendo uma maior vida útil para bateria dos dispositivos. No Brasil, por regulamentação da ANATEL, a banda autorizada para uso do LoRa é a faixa compreendida entre 915 a $928 \mathrm{MHz}$ [Alliance 2015]. O cabeçalho do pacote de cada transmissão pode variar de 2-255 bytes, e a taxa de transferência pode alcaçar até 50Kbps quando aplicado channel aggregation, 
técnica onde são utilizados várias portadoras para aumentar o throughput. A técnica de modulação utilizada é proprietária da Samtech e é no conceito Chirp Spread Spectrum (CSS), que permite a transmissão de dados com relação sinal/ruído extremamente baixa [Alliance 2015].

Dispositivos LoRa podem comunicar-se somente com outros dispositivos LoRa. Para a comunicação com outros dispositivos, especialmente com dispositivos que utilizam a pilha TCP/IP, a Lora Alliance desenvolveu uma tecnologia chamada LoraWAN, que permite essa integração [Alliance 2015].

Uma rede LoraWAN típica é formada por uma topologia estrela, na qual incluem três diferentes tipos de dispositivos: end nodes (ou end devices), gateways e network servers, conforme Figura 1. End nodes possuem aplicações diversas que enviam os dados coletados para gateways utilizando LoRa. Gateways encaminham os pacotes recebidos dos dispositivos para um servidor de rede através de interfaces que utilizam TCP/IP, tipicamente Ethernet ou tecnologias GSM. Consequentemente, gateways são somente relays bidirecionais, ou conversores de protocolo. Como toda a comunicação ocorre de maneira criptografada, os network servers são os responsáveis pela decodificação dos pacotes recebidos. Também são os responsáveis por gerar os pacotes que devem ser enviados de volta para os dispositivos [Augustin et al. 2016].

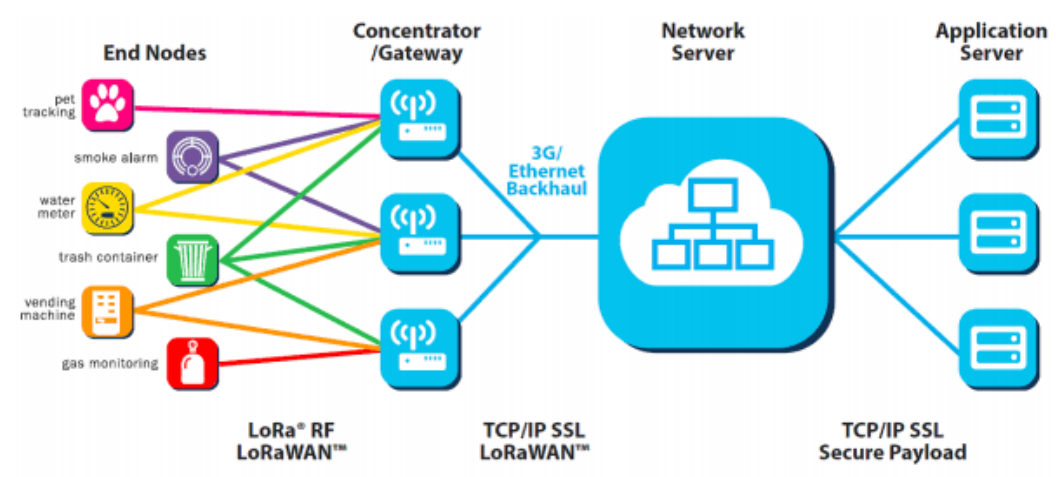

Figura 1. Estrutura de uma rede LoraWAN. Disponível em: https://www.thethingsnetwork.org

Na topologia LoraWAN, os dispositivos podem ser divididos em três classes: A, B e C [Alliance 2015]. Os dispositivos da classe A podem agendar uma transmissão uplink baseado em suas necessidades, com um pequeno jitter. Essa classe de dispositivos permite comunicações bi-direcionais, onde cada envio de transmissão é acompanhado por duas pequenas janelas de recebimentos (downkink). Por consequência, esses dispositivos possuem o menor consumo de energia, porém fornecem menos flexibilidade em transmissões downkink. Os dispositivos classe B possuem mais janelas de comunicação abertas em tempos agendados. Já os dispositivos classe $\mathrm{C}$ possuem janelas de recebimento quase que continuas, porém consomem mais energia.

\section{Cenário}

Para avaliação do LoRa e do LoraWAN, montaram-se dois ambientes: um ambiente para avaliação da distância de comunicação da tecnologia LoRa e outro ambiente para avaliação da conectividade ao LoraWAN. 
Conforme Figura 2, o ambiente de avaliação da distância de comunicação LoRa é composto por dois dispositivos, um realizando a função de cliente, e outro realizando a função de servidor. O dispositivo cliente possui um interruptor responsável por ligar e desligar um LED instalado no servidor. Ambos dispositivos utlizavam a biblioteca RF95 ${ }^{1}$ e foram executados em placas Arduíno $\mathrm{UNO}^{2}$ ligadas a shields Dragino ${ }^{3}$ responsáveis por realizar a comunicação LoRa.

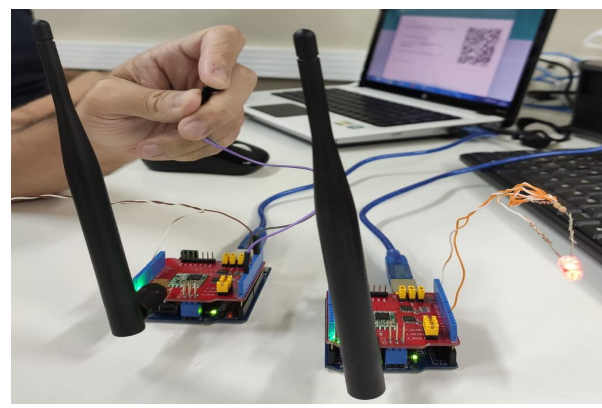

Figura 2. Ambiente de avaliação do LoRa, com dispositivos LoRa ativando e desativando um LED.

O ambiente de avaliação da conectividade ao LoraWAN é composto por um end node e os gateways LoraWAN disponíveis na cidade de Caxias do Sul/RS ${ }^{4}$. O end node é formado pelo conjunto Arduíno mais Dragino, porém executando a biblioteca LMIC ${ }^{5}$. Montou-se também um gateway alternativo, conforme demonstrado na Figura 3, de forma a comparar o desempenho entre este e os demais gateways disponíveis na rede. Esse gateway é formado por um RaspberryPI $3 \mathrm{~B}+{ }^{6}$ ligado a um shield Dragino. No Raspberry foi executado o software single_chan_pkt_fwd ${ }^{7}$, responsável pela execução do gateway.

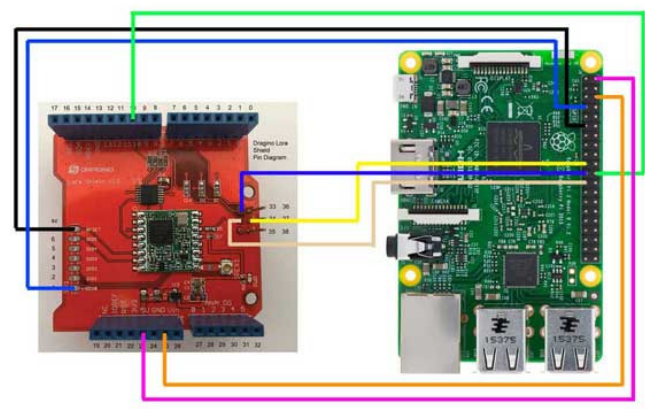

Figura 3. Esquema de ligação do shield Dragino em Raspberry utilizado como gateway LoraWAN. Retirado de http://www.embarcados.com.br.

\section{Avaliação e resultados}

No teste de avaliação da distância de comunicação da tecnologia LoRa, o dispositivo servidor foi mantido em uma das salas do Centro Universitário UniFTEC, enquanto que

\footnotetext{
${ }^{1}$ https://github.com/adafruit/RadioHead

${ }^{2}$ https://www.arduino.cc

${ }^{3}$ https://www.dragino.com

${ }^{4}$ https://www.thethingsnetwork.org/community/caxias-do-sul/

${ }^{5}$ https://github.com/matthijskooijman/arduino-lmic

${ }^{6} \mathrm{https}: / / \mathrm{www}$.raspberrypi.org/products/raspberry-pi-3-model-b-plus

${ }^{7}$ https://github.com/tftelkamp/single_chan_pkt_fwd
} 
o dispositivo cliente movimentou-se pelas redondezas. Neste teste atingiu-se a distância máxima de 240 metros, obtendo cerca $10 \%$ da distância máxima estimada pela LoRa Alliance em cidades. Verificou-se que esse baixo alcance deve-se, entre outros fatores, ao ganho de potência fornecida pela antena padrão da shield Dragino e aos obstáculos (paredes) existentes entre o cliente e o servidor.

Já no teste de avaliação da conectividade ao LoraWAN, não conseguiu-se conectar a nenhuma das antenas públicas disponibilizadas no perímetro urbano de Caxias do Sul/RS. Todavia, houve êxito na conectividade entre o dispositivo e o gateway alternativo montado. De maneira análoga ao teste anterior, o gateway foi mantido na mesma sala, enquanto que o dispositivo foi movimentado pelas redondezas. Além da conectividade alcançada, atingiu-se a distância máxima de comunicação de 90 metros.

Entre as possíveis causas de não conetividade aos gateways LoraWAN disponíveis, pode-se destacar possível uso de frequências fora da faixa 915-928 MHz nos gateways, visto que a informação fornecida pela rede LoraWAN $^{8}$ não permite verificar em qual faixa de frequência cada gateway está operando. Quanto à menor distância de comunicação se comparado ao teste efetuado sobre a rede LoRa "pura", verifica-se que o principal problema está relacionado à aplicação single_chan_pkt_fwd, que permite a comunicação em apenas um dos canais disponíveis na range 915-928MHz.

\section{Conclusão}

Nos ambientes avaliados, verificou-se que a distância de comunicação LoRa atingiu apenas $10 \%$ da distância máxima estimada em documentação e de que foi possível conectarse apenas ao gateway alternativo, não conseguindo conectar-se aos demais gateways existentes na cidade de Caxias do Sul/RS. Esses problemas devem-se especialmente pelo fato de ser uma tecnologia nova e com pouca documentação, especialmente ao que diz respeito à frequência de operação dos próprios gateways. Esses resultados demonstram que, apesar do grande potencial na área em que se propõe a atuar, são necessários mais testes, implementações e pesquisas para que se possa extrair todo o potencial dessa tecnologia.

\section{Referências}

Alliance, L. (2015). Lorawan specification v1.1. Disponível em: https://loraalliance.org/resource-hub/lorawanr-specification-v11. Acesso em 20.nov.2019.

Augustin, A., Yi, J., Clausen, T., and Townsley, W. (2016). A study of lora: Long range \& low power networks for the internet of things. Sensors, 16(9):1466.

Avelar, E., Avelar, L. M., Silva, D. D. P., and Dias, K. L. (2010). Arquitetura de comunicação para cidades inteligentes: Uma proposta heterogénea, extensível e de baixo custo. Universidade Federal de Pernambuco (UFPE), Recife.

Ferrigo, S. F., da Costa, C. A., and da Rosa Righi, R. (2017). Um middleware para gerenciamento de sensores de saude corporais em dispositivos móveis. In $9^{\circ}$ Simposio Brasileiro de Computacao Ubiqua e Pervasiva (SBCUP 2017), volume 9. SBC.

Tomas, G. H. R. P. (2014). Uma arquitetura para cidades inteligentes baseada na internet das coisas. Master's thesis, Universidade Federal de Pernambuco.

\footnotetext{
${ }^{8}$ https://www.thethingsnetwork.org
} 